\title{
Eine neue Biosensor Plattform für den Einsatz in der Tierdiagnostik
}

\author{
Melanie Ewald, Alexander Le Blanc, Valentin Frimmer, Günter Gauglitz \\ Eberhard Karls Universität Tübingen \\ Institut für Physikalische und Theoretische Chemie \\ Auf der Morgenstelle 18 \\ 72076 Tübingen
}

\section{Abstract}

Die Nachfrage nach schnellen und kostengünstigen Analysenmethoden im Bereich der Diagnostik ist in den letzten Jahren zunehmend gestiegen. Dies ist nicht nur in der Humandiagnostik der Fall, sondern auch in der Veterinärdiagnostik. Zum einen sind schnelle Analysenergebnisse wichtig, z.B. um die Ausbreitung von Infektionskrankheiten innerhalb des Tierbestands sowie die Übertragung von Krankheiten zwischen Mensch und Tier (Zoonosen) zu verhindern. Des Weiteren spielt hier auch der wirtschaftliche Faktor eine wesentliche Rolle, wobei Kosten von maximal einem Euro pro Test angestrebt werden.

Für den Nachweis veterinärdiagnostisch relevanter Parameter wurde eine neue Biosensor Plattform entwickelt, die durch ihre direkt optische Detektionseinheit keine Markierungsreagenzien benötigt. Aufgrund ihrer kleinen Dimensionen, ihrer Robustheit und einfachen Handhabung kann die neue Biosensor Plattform auf schnellstem Weg kostengünstig vor Ort eingesetzt werden.

Exemplarisch werden Bioassays vorgestellt, die für die Qualifizierung und Quantifizierung veterinärdiagnostisch relevanter Marker (z.B. Salmonellenantikörper, Chlamydienantikörper, C-reaktives Protein) entwickelt wurden.

\section{Methoden}

\subsection{Biosensoren}

Sensoren bestehen aus einer sensitiven Schicht und einem Transducer. Bei Wechselwirkung der sensitiven Schicht mit dem Analyten wird durch den Transducer die Änderung einer physikalischen Größe in ein messbares Signal umgewandelt. Bei Verwendung von Biosensoren wird typischerweise eine biochemische Wechselwirkung detektiert, z.B. die Wechselwirkung zwischen Enzym und Substrat, Antigen und Antikörper oder komplementären DNA Strängen.

Für den Nachweis veterinärdiagnostisch relevanter Marker mittels Biosensoren muss eine sensitive Schicht entwickelt werden, an die der nachzuweisende Analyt selektiv bindet. Je nach Fragestellung kann der Nachweis relevanter Marker durch zwei grundsätzlich verschiedene Testformate erfolgen: (1) über einen direkten Antigennachweis, wobei das Antigen selbst den Analyten darstellt und ein hochspezifischer Antikörper an die Oberfläche gekoppelt wird und (2) über den Nachweis von spezifischen Antikörpern, die der Wirtsorganismus als Reaktion auf die Infektion gebildet hat. In letzterem Fall dienen Antigenfragmente als Erkennungsstruktur auf der Sensoroberfläche und der Antikörper ist der Analyt.

Zur Detektion der Wechselwirkung zwischen Analyt und sensitiver Schicht wird die Reflektometrische Interferenzspektroskopie (RIfS) [1] sowie die 1-lambda-Reflektometrische Interferenz Sensorik (1-lambdaRIfS) genutzt. Beide Methoden sollen im Folgenden kurz erläutert werden.

\subsection{RIfS}

Bei der RIfS wird ein transparenter Transducer von der Rückseite mit Weißlicht bestrahlt. Die einzelnen Teilstrahlen werden an jeder Grenzfläche gebrochen und reflektiert. Die reflektierten Teilstrahlen interferieren und bilden ein charakteristisches Interferenzspektrum (Bild 1, links).

Bei Anlagerung eines Analyten an die sensitive Schicht, ändert sich der Brechungsindex $\mathrm{n}$ sowie die physikalische Schichtdicke d dieser Schicht, wodurch sich auch der Gangunterschied des Lichtes in dieser Schicht und in den daran reflektierten Teilstrahlen ändert. Dies hat eine Verschiebung des Interferenzspektrums zur Folge (Bild 1, rechts). Die Verschiebung ist proportional dem Produkt aus 
Brechungsindex und physikalischer Schichtdicke (optische Schichtdicke nd). Aufgrund der gegenläufigen Temperaturabhängigkeit beider Größen ist RIfS eine nahezu temperaturunabhängige Methode und dient zur zeitaufgelösten Beobachtung molekularer Wechselwirkungsprozesse.
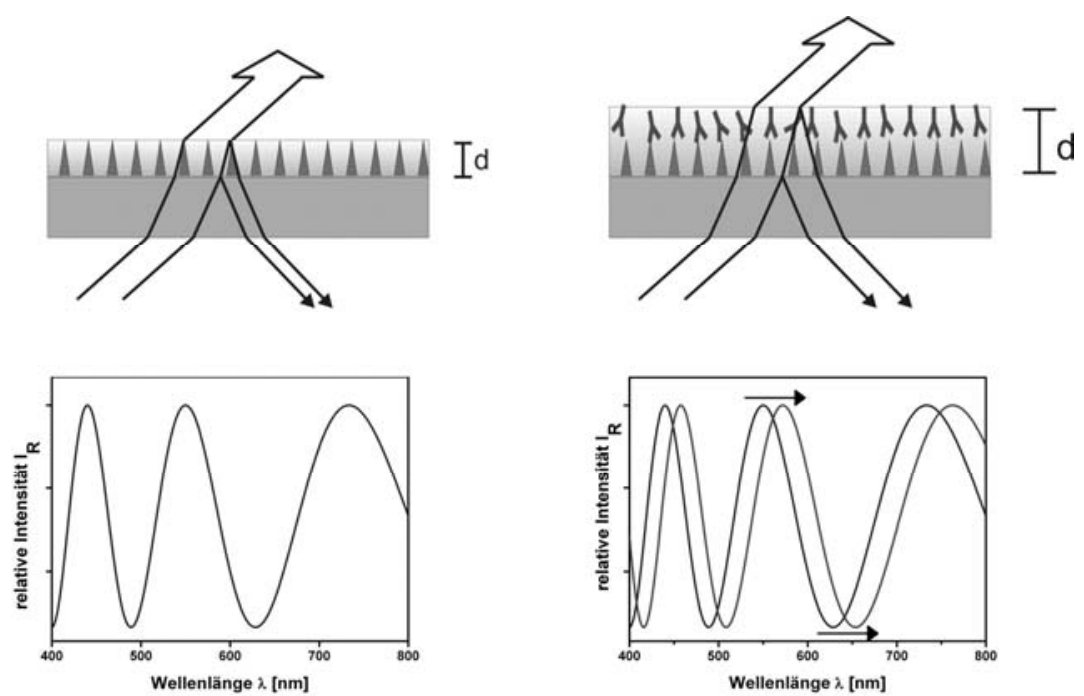

Bild 1 Charakteristisches Interferenzspektrum bei Bestrahlung des Transducers mit Weißlicht (links) und Verschiebung des Interferenzspektrums bei Anlagerung eines Analyten an die sensitive Schicht (rechts).

\subsection{1-lambda-RIfS}

Bei der 1-lambda-Reflektometrischen Interferenzsensorik (1-lambda-RIfS) wird der Transducer von der Rückseite mit einer LED bestrahlt. Während der Inkubation mit dem Analyten wird im Gegensatz zu RIfS die Intensitätsänderung des reflektierten Lichts bei einer Wellenlänge detektiert (Bild 2, links).

Durch die zeitaufgelöste Detektion mit einem Diodenzeilenspektrometer (RIfS) bzw. einer Photodiode (1lambda-RIfS) können Bindungsvorgänge an der Sensoroberfläche im Sekundenbereich beobachtet werden (Bild 2, rechts).
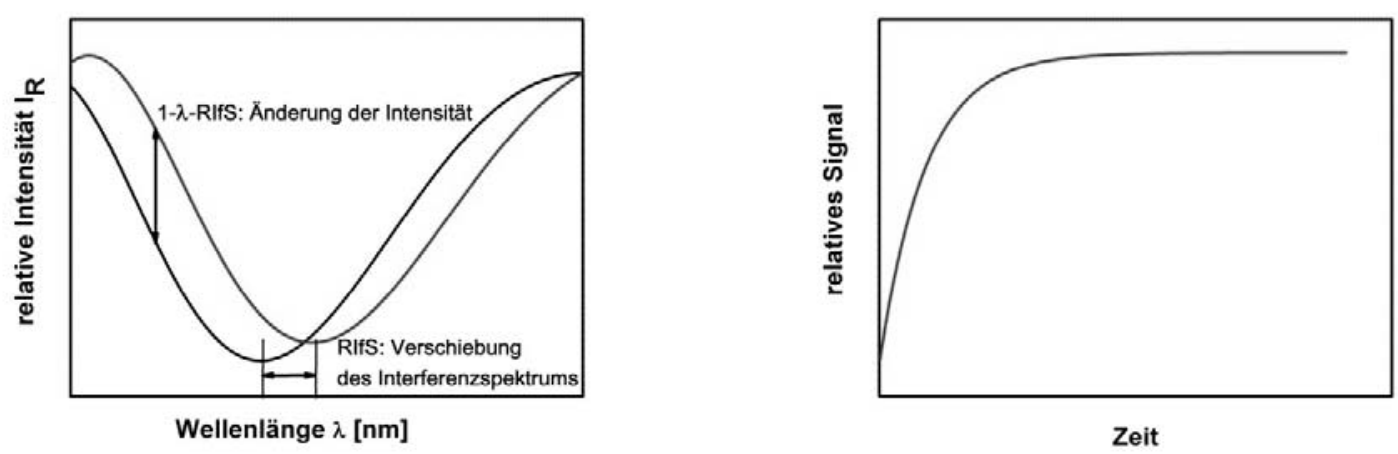

Bild 2 Detektion mit RIfS und 1-lambda-RIfS (links) sowie beispielhaft daraus erhaltenes Bindungssignal (rechts).

\section{Ergebnisse}

\subsection{Nachweis von Infektionen}

Eine mögliche Anwendung der Biosensorplattform ist die Überwachung der Immunantwort bei Nutztieren zum Nachweis von Infektionen. Im Vergleich zu derzeit eingesetzten ELISAs (Enzyme Linked Immunosorbent Assay) kann bei RIfS und 1-lambda-RIfS auf Markierungsreagenzien und damit verbundene Waschschritte vollständig verzichtet werden. Somit können die Kosten pro Test sowie die Analysenzeit deutlich gesenkt werden. Ferner sind durch den portablen Aufbau von 1-lambda-RIfS vor Ort Messungen möglich, sodass auf den Transport entnommener Proben in Laboratorien verzichtet werden kann.

Zur Detektion von Salmonellen- sowie Chlamydieninfektionen wurde mittels RIfS geeignete Assays zum Nachweis der Immunreaktion gegen Salmonellen- bzw. Chlamydienbakterien etabliert. Hierfür wurde das 
Antigen, ein Lipopolysaccharid (LPS), welches auf der Oberfläche der jeweiligen Bakterienzellen vorkommt, auf der Biosensoroberfläche immobilisiert und die hochspezifische Antigen-Antikörper Wechselwirkung zeitaufgelöst verfolgt (Bild 3, links). Nach Etablierung der Assays in Puffer (PBS) wurde als Modellmatrix für Realproben fötales Kälberserum (FCS, fetal calw serum) verwendet und der Sensor damit getestet (Bild 3, rechts).
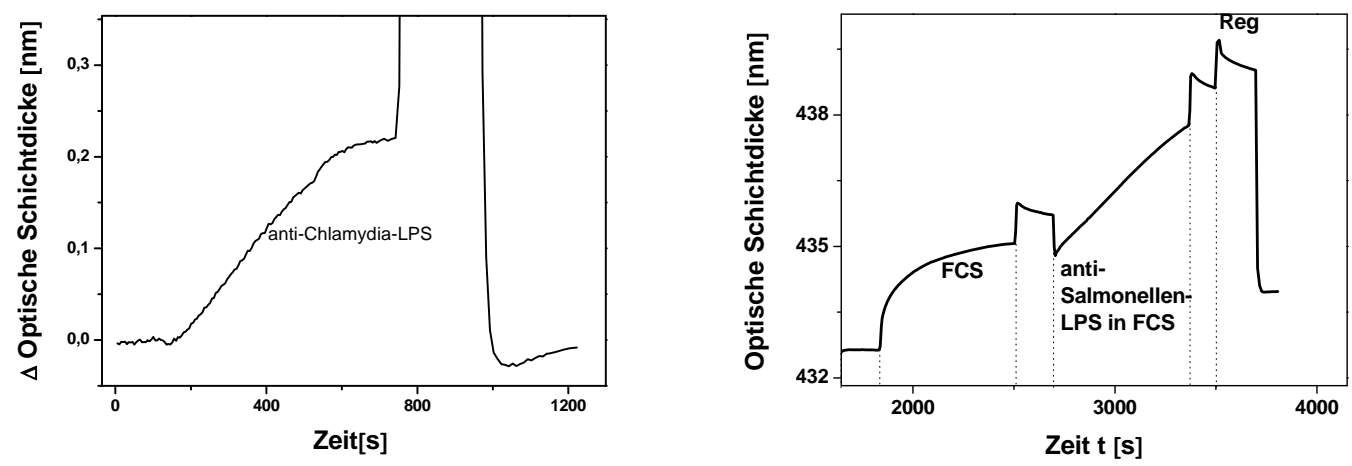

Bild 3 Erhaltenes RIfS-Bindungssignal auf einer Chlamydia-LPS Oberfläche bei Injektion von 20 mg/L ChlamydiaLPS-Antikörper in Puffer (links) und Test einer Salmonellen-LPS-Oberfläche mit der Modellmatrix fötales Kälberserum (FCS) mit darauffolgender Analytinjektion von 10 mg/L LPS-Antikörper in FCS (rechts). Das Signal des Antikörpers wird aufgrund der vorigen Absättigung mit FCS nicht von unspezifischer Wechselwirkung durch Matrixbestandteile überlagert.

In Bild 3 (rechts) wurde gezeigt, dass durch Anpassung des Testverfahrens selbst in unverdünntem Serum als Matrix ein spezifischer Nachweis der Antikörper gegen Salmonellen bzw. Chlamydien durchgeführt werden kann. Nach Optimierung des Transduceraufbaus, der Oberflächenchemie sowie der Fluidik wurde der entwickelte Assay von RIfS auf die neue 1-lambda RIfS Biosensorplattform übertragen.

\subsection{Nachweis von Antigenen}

Für den direkten Antigennachweis ist die stabile Immobilisierung von Antikörpern als sensitive Schicht nötig. Dies kann mittels verschiedener Immobilisierungsstrategien realisiert werden: z.B. a) durch kovalente Anbindung der Antikörper an die Sensoroberfläche oder b) durch die hoch affine Wechselwirkung zwischen Streptavidin und Biotin.

Im Folgenden wird für beide Immobilisierungsstrategien exemplarisch ein Bioassay vorgestellt:

\section{a) Nachweis des Entzündungsmarkers C-reaktives Protein (CRP)}

C-reaktives Protein ist ein Entzündungsmarker, der im Blut vorkommt. Die Konzentration von CRP im Blut eines jungen Erwachsenen liegt bei ca. 1-10 mg/L und kann im Fall einer viralen Infektion auf bis zu 20-40 $\mathrm{mg} / \mathrm{L}$ und bei einer bakteriellen Infektion sogar auf bis zu $500 \mathrm{mg} / \mathrm{L}$ ansteigen [2]. Zur Demonstration der Übertragbarkeit der Technologie auf die Humandiagnostik wurde ein Assay für den Nachweis des Entzündungsmarkers C-reaktives Protein (CRP) etabliert. Für die Bestimmung von CRP aus der Analytlösung (Bild 4) wurden CRP-Antikörper kovalent auf der Biosensoroberfläche immobilisiert.

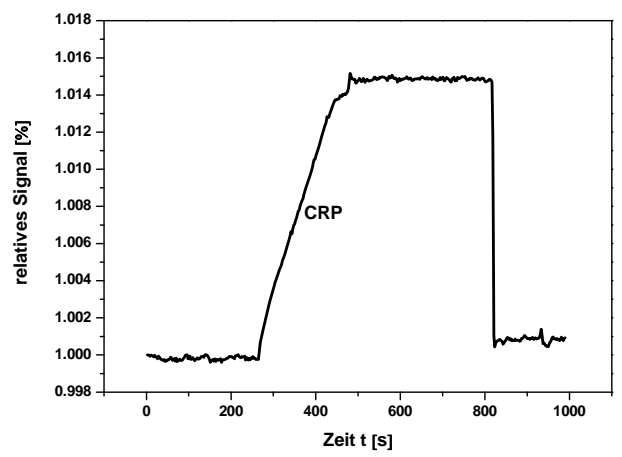

Bild 4 Bindungssignal bei Injektion von 6 mg/L CRP in Puffer über eine anti-CRP-Sensoroberfläche. 


\section{b) Nachweis des Modellantigens Glutathion-S-Transferase (GST)}

Eine weitere Strategie zur Immobilisierung von Antikörpern stellt die Nutzung der hoch affinen Wechselwirkung zwischen Streptavidin und Biotin dar. Streptavidin ist ein tetrameres Protein, dessen Untereinheiten je ein Molekül des Vitamins Biotin (Vitamin H) mit extrem hoher Affinität binden können. Streptavidin kann somit als Bindeglied zwischen einer Biotin-modifizierten Oberfläche und einem Biotinmodifizierten Antikörper dienen. Da eine kovalente Anbindung des Antikörpers an die Oberfläche erfahrungsgemäß zu einer schlechteren Reproduzierbarkeit führt, ist dieses Verfahren von Vorteil. Desweiteren ist es sehr viel einfacher möglich, das Verfahren an andere Antikörper anzupassen.

Soll ein Biotin-modifizierter Antikörper über das Bindeglied Streptavidin auf die Oberfläche gekoppelt werden, so gibt es verschiedene Möglichkeiten [3]:

a) An die Biotin-Oberfläche wird Streptavidin gebunden, gefolgt von dem biotinylierten Antikörper, der an die freien Bindungsstellen des Streptavidins binden soll (Bild 5, links)

b) Der biotinylierte Antikörper wird in homogener Phase mit Streptavidin umgesetzt und das Antikörper-Streptavidin-Konjugat interagiert mit der Biotin-Oberfläche (Bild 5, rechts)
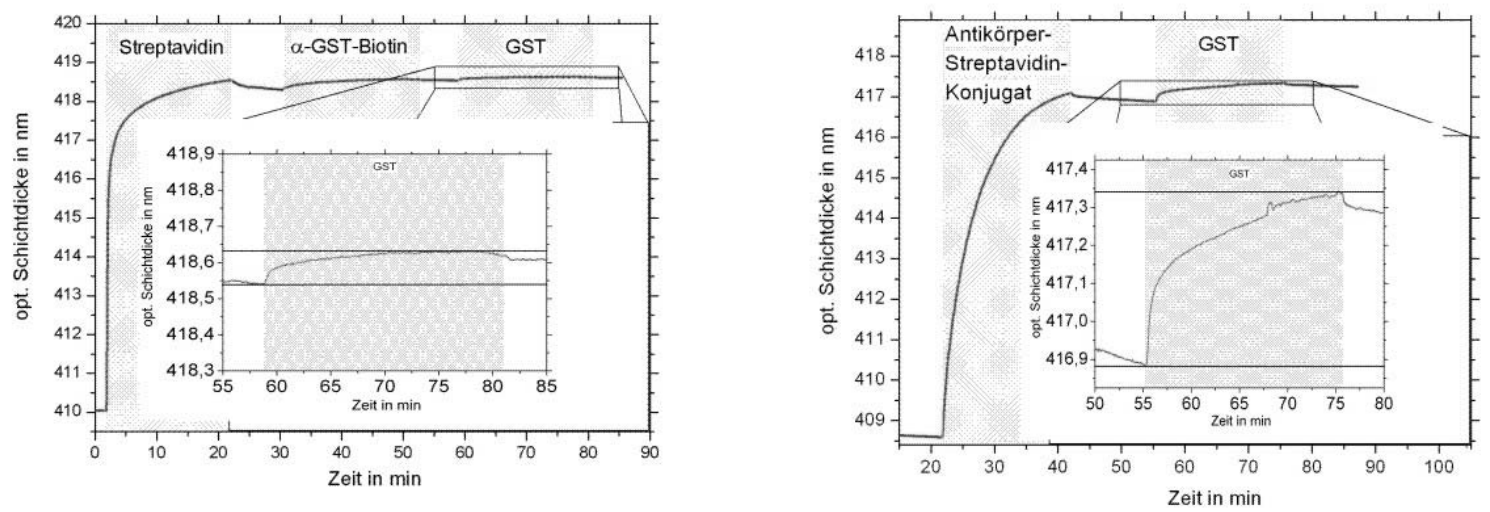

Bild 5 Erhaltene Signale mit RIfS. Links: Bei Zugabe von Streptavidin ist ein starker Anstieg der optischen Schichtdicke zu beobachten. Die geringe Anbindung des biotinylierten Antikörpers im folgenden Schritt macht deutlich, dass nur noch wenige Bindungsstellen für die Anbindung des Antikörpers zur Verfügung stehen. Auch die Anbindung des Modellantigens Glutathion-S-Transferase (GST) fällt bei dieser Strategie sehr gering aus.

Rechts: Wird das Konjugat aus Antikörper und Streptavidin gebildet und diese Lösung über die Biotin-Oberfläche geleitet, so ist eine hohe Belegung mit Antikörpern $\mathrm{zu}$ beobachten, was durch einen gut messbaren Schichtdickenzuwachs bei Injektion des Modellantigens GST deutlich wird.

\section{Ausblick}

Es wurden mehrere Bioassays für den Nachweis veterinärdiagnostisch relevanter Marker entwickelt und erfolgreich auf die neue Biosensor Plattform übertragen. Für Multiparametermessungen wird ein geeigneter Laboraufbau konzipiert, sodass mehrere Marker gleichzeitig aus einer Probe bestimmt werden können. Hierfür sind die Harmonisierung der Assaybedingungen sowie der Oberflächenchemie erforderlich.

\section{Literatur}

[1] G. Proll, G. Markovic, L. Steinle, G. Gauglitz (2009) Reflectometric Interference Spectroscopy. Methods Mol Biol. 503(1):167-78.

[2] C. Albrecht, N. Kaeppel, G. Gauglitz (2008) Two immunoassay formats for fully automated CRP detection in human serum. Anal Bioanal Chem 391(5):1845-1852.

[3] A. F. Le Blanc, M. Ewald, G. Proll, J. Sindlinger, G. Gauglitz (2010), A review on reflectometric interference spectroscopy with antibody immobilization in animal diagnostics, GIT Labor-Fachzeitschrift, 54(2):90-93. 